\title{
Fotobiomodulação empregada no tratamento e/ou prevenção da fadiga muscular em humanos: Direcionamento para a empregabilidade clínica
}

\author{
Photobiomodulation used in the treatment and / or prevention of muscle fatigue in humans:
}

Direction for clinical employability

Fotobiomodulación utilizada en el tratamiento y / o prevención de la fatiga muscular en humanos:

dirección para la empleabilidad clínica

Recebido: 22/03/2021 | Revisado: 28/03/2021 | Aceito: 28/03/2021 | Publicado: 09/04/2021

\author{
David Ribeiro Costa \\ ORCID: https://orcid.org/0000-0002-4188-1321 \\ Centro de pesquisa Avançada em Fototerapia, Brasil \\ E-mail: profdavid8@gmail.com.br \\ Carolina Alves Delpasso \\ ORCID: https://orcid.org/0000-0002-4700-5883 \\ Centro de pesquisa Avançada em Fototerapia, Brasil \\ E-mail: delpassoc@gmail.com \\ Luana Aparecida Pilato Ribeiro \\ ORCID: https://orcid.org/0000-0002-2317-8835 \\ Centro de pesquisa Avançada em Fototerapia, Brasil \\ E-mail: luana_pilato@yahoo.com.br \\ Thiago dos Santos Maciel \\ ORCID: https://orcid.org/0000-0002-7022-2309 \\ Universidade Federal do Amazonas, Brasil \\ E-mail: thiagomaciel@ufam.edu.br \\ Davidson Ribeiro Costa \\ ORCID: https://orcid.org/0000-0002-9750-5675 \\ Centro de pesquisa Avançada em Fototerapia, Brasil \\ E-mail: dnribcosta@hotmail.com.br
}

\begin{abstract}
Resumo
O objetivo do presente trabalho foi realizar um levantamento dos principais parâmetros de aplicação da fotobiomodulação proveniente da Terapia a Laser de Baixo Intensidade (TLBI) e/ou da terapia com diodos emissores de luz (LEDT) empregada sobre a FM, além de propor sua empregabilidade clínica por meio de protocolos que apresente evidências científicas. Foi realizado um levantamento bibliográfico nas bases de dados do PUBMED, utilizando as palavras-chaves disponíveis nos DESCs: "Photobiomodulation Therapy", "PBTM", "Low-Level Light Therapy", "LLLT", "Light-Emitting Diode Therapy”, LEDT", "Phototherapy”, "Exercise”, "Fatigue”, "Performance”, "sport" em diferentes combinações e associadas aos descritores booleanos. Foram considerados os seguintes critérios de inclusão: ensaios clínicos controlados e randomizados, publicados na língua inglesa, período de publicação entre os anos 2017 a 2020. Com isso, foi feita a leitura aprofundada dos artigos e mediante a aplicação dos critérios de inclusão e exclusão, restaram somente dez artigos publicados. A terapia de fotobiomodulação realizada por meio da utilização da TLBI e LEDT é capaz de atrasar o processo de FM e consequentemente aumentar a performance. Contudo os parâmetros ideais de aplicação dessa ferramenta ainda continuam demostrando muitas variáveis. Exceto pelo comprimento de onda, que é o único parâmetro de aplicação que demostra uma tendência.
\end{abstract}

Palavras-chave: Terapia a laser; Fadiga muscular; Terapia com luz de baixa intensidade.

\begin{abstract}
The objective of the present work was to survey the main application parameters of photobiomodulation from LowLevel Light Therapy (LLLT) and / or diode therapy. light emitters (LEDT) used on FM, in addition to proposing their clinical employability through protocols that present scientific evidence. A bibliographic survey was carried out in the PUBMED databases, using the keywords available in the DESCs: "Photobiomodulation Therapy", "PBTM", "LowLevel Light "LLLT", "Light-Emitting Diode Therapy", "LEDT","Phototherapy","Exercise","Fatigue","Performance","sport" in different combinations and associated with Boolean descriptors. The following inclusion criteria were considered: controlled and randomized clinical trials, published in English, publication period between the years 2017 to 2020 After this procedure, all abstracts were read, in which fifteen articles were selected. In the last stage, the articles were read in depth and, by applying the inclusion and exclusion criteria, only ten published articles remained. Photobiomodulation therapy performed using TLBI and
\end{abstract}


LEDT is able to delay the FM process and consequently increase performance. However, the ideal application parameters of this tool still show many variables. Except for the wavelength, which is the only application parameter that shows a trend.

Keywords: Laser therapy; Muscle fatigue; Low-level light therapy.

\section{Resumen}

El objetivo del presente trabajo fue realizar un relevamiento de los principales parámetros de aplicación de la fotobiomodulación de la Terapia Láser de Baja Intensidad (TLBI) y/ la terapia con diodos emisores de luz (LEDT) utilizados en FM, además de proponer su empleabilidad. protocolos que presentan evidencia científica. Se realizó un relevamiento bibliográfico en las bases de datos PUBMED, utilizando las palabras clave disponibles en los DESC: "Terapia de fotobiomodulación", "PBTM", "Terapia de luz de bajo nivel", "LLLT", "Terapia con diodos emisores de luz", "LEDT", "Fototerapia", "Ejercicio", "Fatiga", "Rendimiento", "Deporte" en diferentes combinaciones y asociadas a descriptores booleanos. Se consideraron los siguientes criterios de inclusión: ensayos clínicos controlados y aleatorizados, publicados en idioma inglés, período de publicación entre los años 2017 a 2020. Con esto, los artículos fueron leídos en profundidad y a través de los criterios de inclusión y exclusión solo quedaron diez artículos publicados. La terapia de fotobiomodulación realizada con TLBI y LEDT puede retrasar el proceso de FM y, en consecuencia, aumentar el rendimiento. Sin embargo, los parámetros de aplicación ideales de esta herramienta aún muestran muchas variables. Excepto por la longitud de onda, que es el único parámetro de aplicación que muestra una tendencia.

Palabras clave: Terapia por láser; Fatiga muscular; Terapia por luz de baja intensidad.

\section{Introdução}

A Fadiga Muscular (FM) é um processo complexo e multifacetado que envolve elementos fisiológicos e biomecânicos (Leal Júnior et al., 2010). Normalmente é caracterizada pela diminuição inconsciente da capacidade do organismo de gerar ou manter a força muscular em determinada tarefa (Boccia et al., 2016). Essa diminuição, funciona como um processo de defesa do organismo, impedindo que as reservas de energia do corpo se esgotem (Costa et al., 2015).

O processo de FM além de levar a um declínio no desempenho físico, contribui de maneira efetiva no acometimento de lesões musculares (Tomazini et al., 2019), pois a sua instauração pode prejudicar o ato muscular e a sustentação dos movimentos cinéticos, além de sobrecarregar outros grupos musculares (Miranda et al., 2017).

Todavia, nas últimas décadas a FM vem sendo objeto de estudo, em inúmeras investigações sobre condições que podem levar à FM, recuperação e/ou métodos de como evitá-la ou retardá-la, são assuntos frequentemente abordados na literatura (Leal Júnior et al., 2010; Miranda et al., 2017; Costa et al., 2021).

Entretanto, uma ferramenta tem se destacando por sua versatilidade e resultados promissores na diminuição de indicies de FM e no reparo de danos musculares causados por exercícios extenuantes (Dorneles et al., 2019). Esta ferramenta é conhecida como fotobiomodulação (Anders et al., 2019).

A fotobiomodulação, proveniente da Terapia a Laser de Baixo Intensidade (TLBI) e/ou da terapia com diodos emissores de luz (LEDT), é considerada uma terapia não invasiva, e estudos têm sugeridos efeitos positivos na diminuição da dor, inflamação e na prevenção da FM, e consequentemente, no aumento da performance muscular (Anders et al., 2019; Dorneles et al., 2019).

Dentre os mecanismos de ação da fotobiomodulação sobre os tecidos biológicos estão relacionados à absorção de luz por cromóforos nas células, como o citocromo c oxidase (Cox) proposto como o principal agente absorvedor da luz. A enzima Cox, unidade IV na cadeia respiratória mitocondrial, desencadeia uma variedade de efeitos após a absorção da luz (Ferraresi et al., 2016; Costa et al., 2017; Pessoa et al., 2018).

Entre esses efeitos, a literatura destaca o aumento da síntese de adenosina trifosfato (ATP) e modulações nas taxas de síntese de DNA e RNA, que por sua vez afetam a proliferação celular e expressão gênica relacionadas a várias vias celulares, como mitose, apoptose, inflamação e metabolismo da energia mitocondrial, o que pode estar relacionado aos efeitos positivos observados no tratamento e prevenção da FM (Ferraresi et al., 2016; Costa et al., 2017). 
Contudo, hoje a grande pergunta que se faz sobre a terapia de fotobiomodulação não é mais sobre a sua eficácia em retardar o processo de FM, mas sim, quais são os parâmetros de aplicação que possa otimizar esses efeitos? Já que essa ferramenta possibilita inúmeras configurações de aplicações clínicas (Ferraresi et al., 2015; De Oliveira et al., 2017).

Segundo De Oliveira et al. (2017) existe uma grande variabilidade de parâmetros utilizados nos estudos, o que dificulta a interpretação dos resultados e da utilização dessa ferramenta no meio clínico.

Portanto, o objetivo do presente trabalho é fazer um levantamento dos principais parâmetros de aplicação da fotobiomodulação empregada sobre a FM, além de propor sua empregabilidade clínica por meio de protocolos que apresente evidências científicas.

\section{Metodologia}

O presente trabalho foi realizado por meio de uma revisão sistemática, na qual foram seguidas as recomendações do Preferred Reporting Items for Systematic Review and Meta-Analyses (PRISMA) (Moher et al., 2009). Para tal, foi realizado um levantamento bibliográfico nas bases de dados do PUBMED, utilizando as palavras-chaves disponíveis nos DESCs: "Photobiomodulation Therapy", "PBTM", "Low-Level Light Therapy", "LLLT", "Light-Emitting Diode Therapy", LEDT", "Phototherapy", "Exercise", "Fatigue", "Performance", "sport" em diferentes combinações e associadas aos descritores booleanos.

Foram considerados os seguintes critérios de inclusão: ensaios clínicos controlados e randomizados, publicados na língua inglesa, período de publicação entre os anos 2017 e 2020, que abordassem uso da fotobiomodulação no tratamento da FM em humanos, pontuação mínima de 5 no escore da avaliação da qualidade metodológica da Escala PEDro. Como critérios de exclusão estão experimentos realizados em modelo animal, em indivíduos que possuíssem algum tipo de doença, e aqueles publicados fora do período preconizado, escore baixo e trabalhos com metodologia e/ou conclusões insatisfatórias.

Estudos em potenciais foram avaliadas por dois autores que examinaram independentemente todos os títulos e resumos. Discordâncias entre autores da revisão foram resolvidos com decisão do terceiro autor.

Dos artigos selecionados, foram tabulados os seguintes itens: autor/ ano, população, comprimento de onda, dose de energia total aplica no musculo, intervalo da aplicação até o momento dos exercícios, musculo irradiado, atividade física testada, e os resultados obtidos.

\section{Resultados e Discussão}

O fluxograma 1 (Figura 1) apresenta os resultados referentes aos artigos pesquisados no período estabelecido. Foram encontrados trinta e dois artigos na PubMed. Com a verificação dos títulos foram selecionados vinte cinco artigos. Após esse procedimento, foi feita a leitura de todos os resumos, onde quinze artigos foram selecionados. Na última etapa foi feita a leitura aprofundada dos artigos e mediante a aplicação dos critérios de inclusão e exclusão, restaram somente dez artigos publicados. 
Figura 1. Fluxograma de seleção dos estudos utilizados na revisão de literatura sobre os efeitos da PBMT sobre a FM.

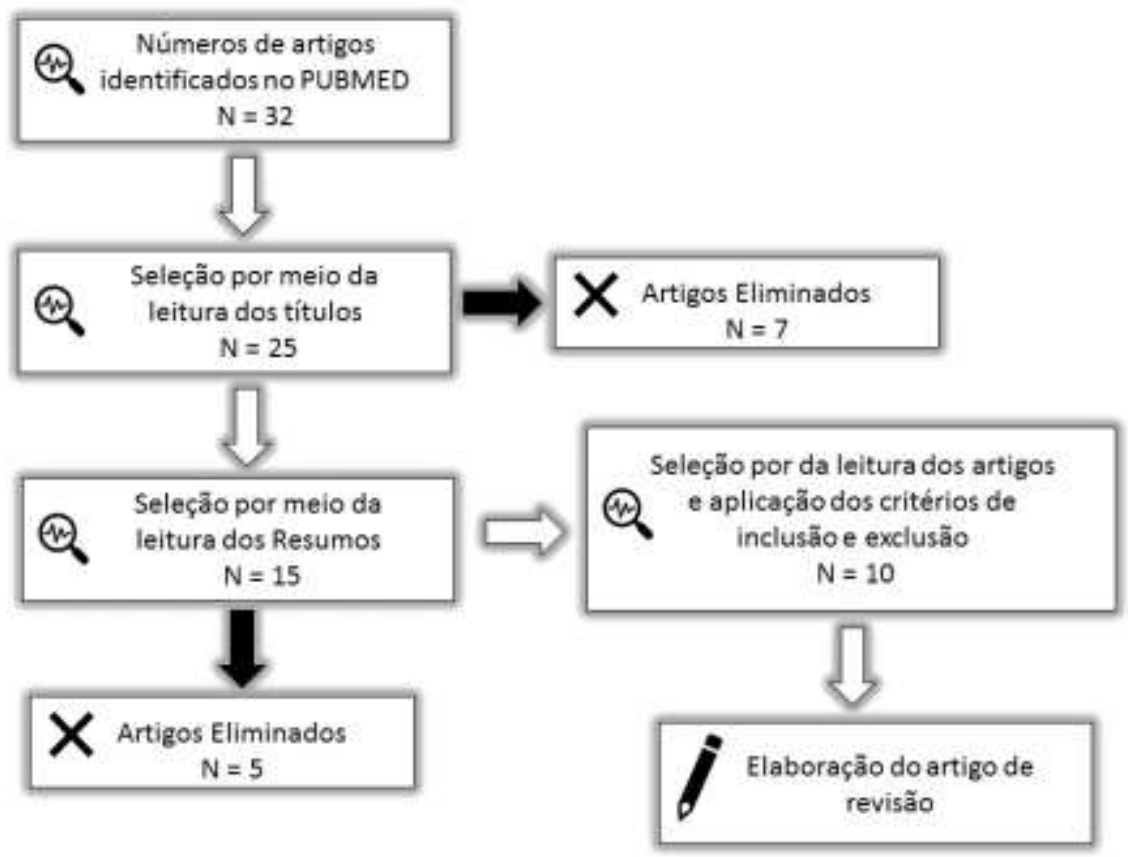

Fonte: Autores (2021).

A Tabela 1 apresenta as características dos dez artigos selecionados para esta revisão, os quais foram organizados da seguinte forma: autor/ ano, população, comprimento de onda, dose de energia total aplica no musculo, intervalo do momento da aplicação até a realização dos testes, musculo irradiado, atividade física testada, e o efeito sobre a FM. Os itens que compõem a tabela foram selecionados para a identificação das pesquisas (autor/ano, população, musculo irradiado e teste utilizado), parâmetros que se mostraram essenciais para os resultados (comprimento de onda, dose de energia total aplica no musculo e o intervalo da aplicação até o momento dos exercícios) e por fim o resultado (efeito sobre a FM).

Tabela 1. características dos artigos selecionados.

\begin{tabular}{|c|c|c|c|c|c|c|c|}
\hline Autor/ano & população & $\lambda(\mathbf{n m})$ & $\begin{array}{l}\text { Energia } \\
\text { Total (J) }\end{array}$ & $\begin{array}{l}\text { Intervalo } \\
\text { (min) }\end{array}$ & Músculo & Atividade & Efeito \\
\hline $\begin{array}{l}\text { ORSSATO et } \\
\text { al., } 2019\end{array}$ & $\begin{array}{l}\text { Atletas de } \\
\text { judô }\end{array}$ & $\begin{array}{c}670, \\
850, \\
880 \text { e } \\
950\end{array}$ & $\begin{array}{c}60,120 \mathrm{e} \\
240\end{array}$ & NR & $\begin{array}{l}\text { Quadríceps, } \\
\text { Isquiotibiais e } \\
\text { gastrocnêmico }\end{array}$ & Saltos & + \\
\hline $\begin{array}{l}\text { DORNNELES } \\
\text { et al., } 2019\end{array}$ & $\begin{array}{l}\text { Jogadores } \\
\text { de futebol }\end{array}$ & 880 & 300 & NR & $\begin{array}{l}\text { Quadríceps, } \\
\text { Isquiotibiais e } \\
\text { gastrocnêmico }\end{array}$ & $\begin{array}{l}\text { Extensão do } \\
\text { joelho } \\
\text { (Dinamômetro } \\
\text { Isocinético) }\end{array}$ & + \\
\hline $\begin{array}{l}\text { TOMAZINI et } \\
\text { al., } 2019\end{array}$ & $\begin{array}{l}\text { Jogadores } \\
\text { de futebol }\end{array}$ & 810 & $\begin{array}{c}100,300 \mathrm{e} \\
450\end{array}$ & NR & $\begin{array}{l}\text { Quadríceps, } \\
\text { Isquiotibiais e } \\
\text { gastrocnêmico }\end{array}$ & $\begin{array}{l}\text { Teste de } \\
\text { corrida }\end{array}$ & + \\
\hline $\begin{array}{c}\text { Da CUNHA et } \\
\text { al., } 2019\end{array}$ & $\begin{array}{l}\text { Atletas de } \\
\text { voleibol }\end{array}$ & 850 & 36 & & Quadríceps & Saltos & + \\
\hline $\begin{array}{c}\text { DELLAGRANA } \\
\text { et al., } 2018\end{array}$ & Corredores & $\begin{array}{c}670, \\
850, \\
880 \text { e } \\
950\end{array}$ & 840 e 420 & NR & $\begin{array}{l}\text { Quadríceps, } \\
\text { Isquiotibiais e } \\
\text { gastrocnêmico }\end{array}$ & $\begin{array}{l}\text { Teste de } \\
\text { corrida }\end{array}$ & + \\
\hline
\end{tabular}




\begin{tabular}{|c|c|c|c|c|c|c|c|}
\hline $\begin{array}{l}\text { ROSSATO et } \\
\text { al., } 2018\end{array}$ & Corredores & $\begin{array}{c}670, \\
850, \\
880 \text { e } \\
950\end{array}$ & 270 & 0 e 360 & Quadríceps & $\begin{array}{c}\text { Extensão do } \\
\text { joelho } \\
\text { (Dinamômetro } \\
\text { Isocinético) }\end{array}$ & + \\
\hline $\begin{array}{l}\text { MIRANDA et } \\
\text { al., } 2017\end{array}$ & Corredores & $\begin{array}{c}640, \\
875 \mathrm{e} \\
905\end{array}$ & 510 & NR & $\begin{array}{l}\text { Quadríceps, } \\
\text { Isquiotibiais e } \\
\text { gastrocnêmico }\end{array}$ & $\begin{array}{l}\text { Teste de } \\
\text { corrida }\end{array}$ & + \\
\hline $\begin{array}{l}\text { LANFERDINI } \\
\text { et al., } 2017\end{array}$ & $\begin{array}{l}\text { Atletas de } \\
\text { ciclismo }\end{array}$ & 810 & $\begin{array}{c}135,270 \mathrm{e} \\
450\end{array}$ & NR & Quadríceps & $\begin{array}{l}\text { Teste de } \\
\text { ciclismo }\end{array}$ & + \\
\hline $\begin{array}{c}\text { De OLIVEIRA } \\
\text { et al., } 2017\end{array}$ & $\begin{array}{l}\text { Jogadores } \\
\text { de Futebol }\end{array}$ & 810 & 300 & NR & Quadríceps & $\begin{array}{l}\text { Extensão do } \\
\text { joelho } \\
\text { (Dinamômetro } \\
\text { Isocinético) }\end{array}$ & + \\
\hline $\begin{array}{c}\text { HEMMINGS et } \\
\text { al., } 2016\end{array}$ & Corredores & $\begin{array}{l}850 \\
660\end{array}$ & $720,180,45$ & NR & Quadríceps & $\begin{array}{l}\text { Extensão do } \\
\text { joelho } \\
\text { (Dinamômetro } \\
\text { Isocinético) }\end{array}$ & + \\
\hline
\end{tabular}

Legenda: $\lambda(\mathrm{nm})=$ Comprimento de onda em nanômetro; NR=não relatado pelo estudo; += Efeito positivo; $0=$ efeito nulo. Fonte: Autores.

Com os resultados levantados foi possível constatar que todos os artigos selecionados identificaram em suas metodologias o comprimento de onda utilizado.

Em seu estudo De Oliveira et al. (2017), relata que a terapia de fotobiomodulação exibe um padrão bifásico doseresposta, o que significa que doses intermediárias localizadas dentro de uma "janela terapêutica" estimula os tecidos biológicos, enquanto doses abaixo ou acima dessa janela não desencadeia nenhum efeito terapêutico. Para Rosssato et al. (2018) os efeitos fisiológicos e terapêuticos fornecidos pela terapia de fotobiomodulação são devidos a fatores fotofísicos e eventos fotoquímicos gerados após a absorção da energia luminosa pelos cromóforos específicos mitocôndrias (especialmente citocromo c oxidase, unidade IV de cadeia respiratório no sistema mitocondrial). Assim, aparelhos de TLBI ou LEDT quando utilizam comprimentos de ondas de $650 \mathrm{~nm}$ a $1200 \mathrm{mn}$ tem a maior chance de estimular tecidos biológicos pois esses valores correspondem a " janela terapêutica ". O mecanismo de ação da fotobiomodulação pode ser observada na Fgura 2. 
Figura 2. Ação da fotobiomodulação nos tecidos.

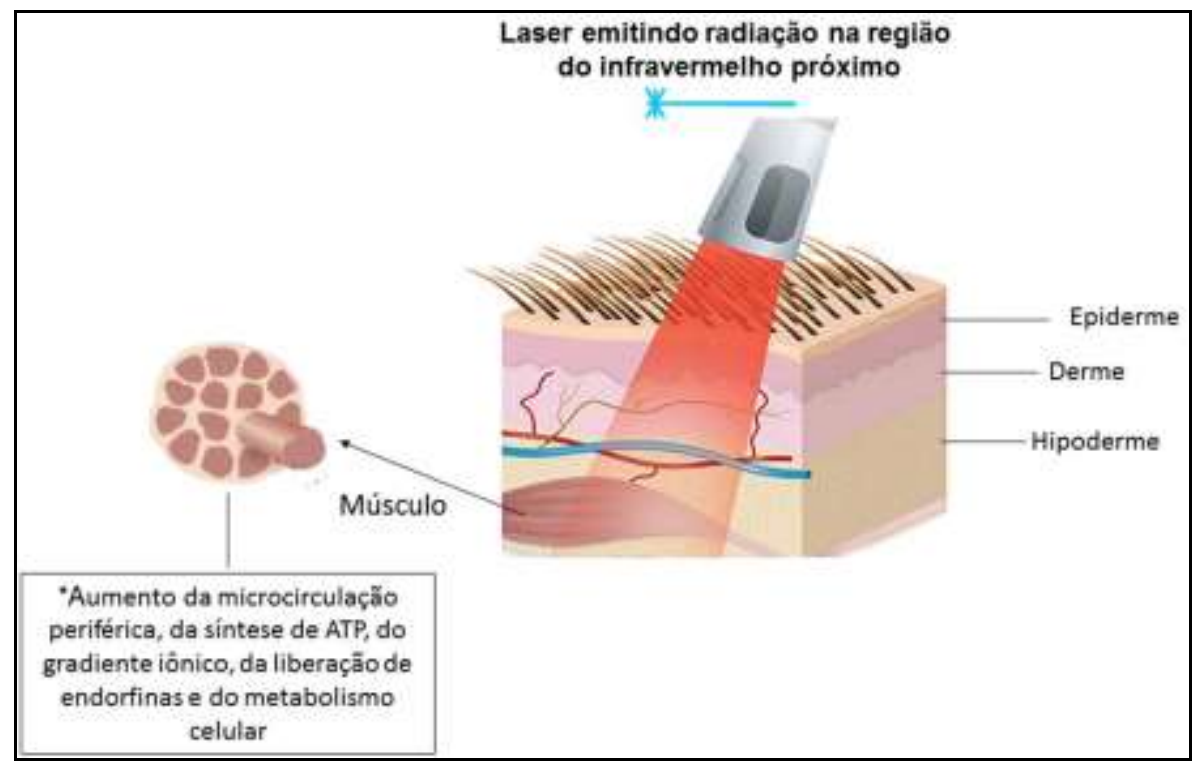

Fonte: Autores (2021).

Em cinco estudos foram encontrados combinação de diferentes comprimentos de onda em uma mesma aplicação. Três apresentaram as mesmas combinações 950,880, 850 e $670 \mathrm{~nm}$ (7,16,17). Miranda et al. (2017), foi o único que combinou os comprimentos de ondas: 905,875 e $640 \mathrm{~nm}$. Hemmings et al. (2016), combinaram apenas 2 comprimentos de onda que foram 850 e $650 \mathrm{~nm}$.

Observou-se que essas combinações de comprimento de onda só foram possíveis por meio da utilização de um cluster de aplicação, esse equipamento consegue misturar Lasers e Leds de diferentes combinações de comprimentos de onda (dependendo do fabricante) (Figura 3).

Figura 3. Diferentes Clusters de aplicação de PBMT (Lasers e LEDs combinados).

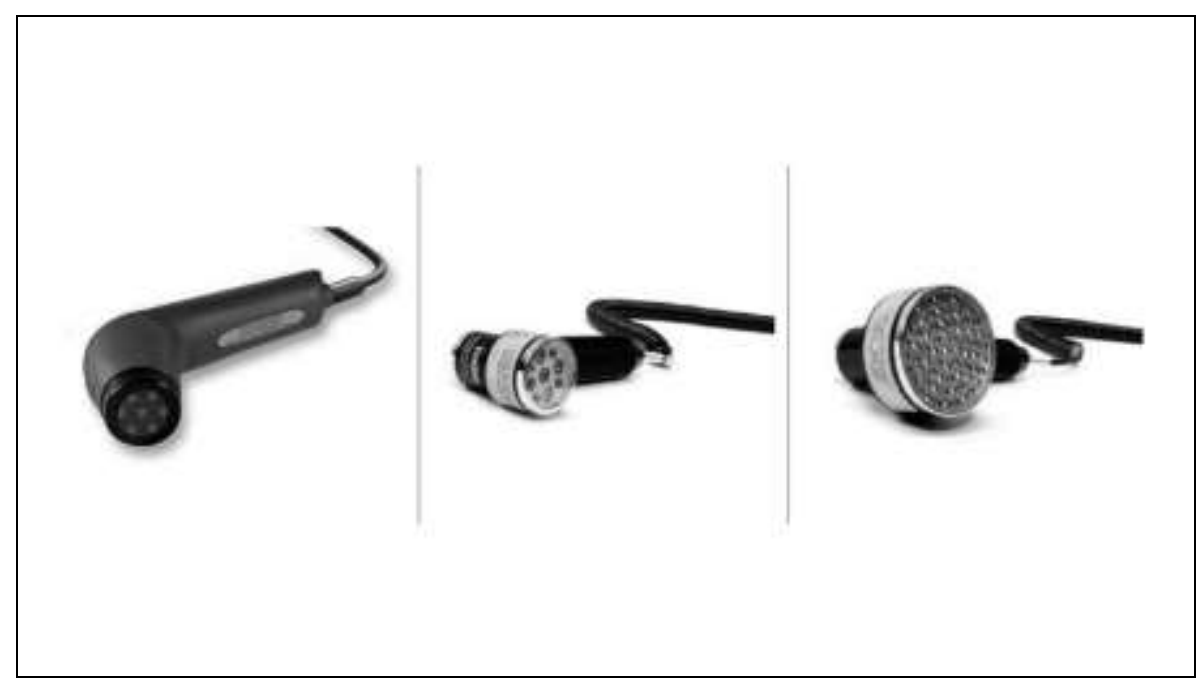

Fonte: Autores (2021).

O primeiro trabalho a combinar diferentes comprimentos de ondas e investigar sua a ação na FM foi AlbuquerquePontes et al. (2014), que teve como objetivo avaliar os efeitos de diferentes doses e comprimentos de onda, sobre a modulação energética do citocromo c oxidase em modelo animal. 
Os resultados obtidos, foram as primeiras indicações que o uso combinado de diferentes comprimentos de onda ao mesmo tempo pode melhorar os efeitos da terapia de fotobiomodulação, em relação ao desempenho muscular retardando o processo da FM.

Entretanto, ao longo de décadas estudos que não combinaram diferentes comprimentos de ondas, também obtiveram resultados positivos. Em comum entre eles, além dos resultados, foi a utilização de comprimento de onda na faixa do infravermelho.

Em nossa revisão as faixas espectrais no infravermelho variaram de $810 \mathrm{~nm}$ a $950 \mathrm{~nm}$ que correspondem a "janela terapêutica" citada por Oliveira et al. (2017) e Rossato et al. (2018).

Leal Jr et al., (2010) relata em seu estudo que a irradiação no infravermelho possui um potencial maior de penetração nos tecidos biológicos. Isso se deve ao fato de que a luz nesta região não é completamente absorvida nas primeiras camadas do tecido biológico.

Contudo podemos identificar que a investigação, enquanto, ao comprimento de onda para parâmetro de aplicação da terapia de fotobiomodulação no tratamento da FM já está bem elucidado pela literatura, porém isso não acontece em outros parâmetros.

O segundo item analisado foi a dose total(J) aplicada no tecido. Todos os estudos selecionados apresentaram esse parâmetro em suas metodologias. Em nosso levantamento, pode se observar que as dose total (J) utilizadas variaram de 38J a 840J de energia total aplicada sobre o musculo, porém, muitos estudos utilizaram grupos musculares diferentes. Entretanto, duas pesquisas investigaram diferentes dose aplicadas em um mesmo músculo.

Hemmings et al. (2016) em seu estudo teve como objetivo comparar o efeito da aplicação da terapia de fotobiomodulação com 3 diferentes doses (720, 180 e 45 J) no musculo quadríceps em corredores. Participaram de seu estudo 34 atletas amadores de ambos os sexos, onde todos passaram pelos grupos (Placebo, 720J,180J e 45J) com um intervalo de um mês de diferença entre as seções de teste. Os parâmetros avaliatórios escolhidos foram a análise do lactato sanguíneo pré e pós exercício e a utilização do dinamômetro isocinético (para avaliar a quantidade de repetições realizada no exercício). Por meio dos resultados foi possível observar que houve um aumento significativo no número de repetições realizadas no grupo 180J(p $=0,023)$ e $720 \mathrm{~J}(\mathrm{p}=0,004)$ de irradiação, quando comparado com o grupo placebo. Em relação ao lactato sanguíneo não houve diferença entre os grupos. Contudo os autores concluíram que as dose total(J) de 180J e 720J de energia total aplicada nos músculos quadríceps foram as que conseguiram otimizar os efeitos da terapia de fotobiomodulação.

Resultados diferentes foram encontrados na pesquisa de Lanferdini et al. (2017), onde testou o efeito da aplicação da terapia de fotobiomodulação com 3 diferentes doses no musculo quadríceps sobre a cinética do VO2 de atletas durante testes de ciclismo. As doses testadas foram 135, 270 e 405J. Participaram de seu estudo 22 ciclistas que passaram por todos os grupos de tratamento (placebo, 135J, 207J e 405J). Os parâmetros avaliatórios escolhidos foram um teste de ciclagem incremental até a exaustão para determinar captação máxima de oxigênio (VO2MAX) e potência máxima (POMAX). Os resultados mostraram que a irradiação da terapia de fotobiomodulação foi capaz de retardar o processo de FM e assim aumenta a performance muscular. Contudo não foi encontrado diferenças significativas entre as doses totais de energia testadas.

Denota-se que esse parâmetro ainda não está totalmente elucidado pela literatura devido a imensa dificuldade em estabelece padrões em sua utilização e resultados conflitantes.

O terceiro parâmetro de aplicação analisado foi o intervalo do momento da aplicação a até a realização do teste, o chamado tempo de resposta do efeito da fotobiomodulação.

O primeiro estudo que identificou esse parâmetro como essencial foi Ferraresi et al. (2015), que teve como objetivo, determinar a resposta temporal da terapia de fotobiomodulação no aumento da sínteses de ATP e consequentemente retardar o processo de FM. Em sua metodologia em modelo animal foi utilizado 50 camundongos (sexo masculino), no qual, foram 
testados 4 intervalos diferentes (5min, 3h, 6h e 24h) após aplicação da terapia de fotobiomodulação. Os resultados mostraram que o pico de performance aconteceu com a adoção do intervalo de 6h. Contudo os pesquisadores concluíram que o tempo de respostas em camundongos provavelmente é diferente em humanos.

Em nosso levantamento apenas Rossato et al. (2018) identificou esse parâmetro em sua metodologia, e justamente com o intuito identificar os efeitos de duas respostas de tempo diferentes (0min e 6h). Participaram 16 voluntários do sexo masculino. Os parâmetros avaliatórios escolhidos foram a análise da ativação elétrica muscular por meio da utilização da eletromiografia de superfície e análise de força máxima e resistência muscular mediante a testes no diâmetro isocinético. Com os resultados obtidos, foi possível observar que a utilização do intervalo de 0 min possibilitou a otimização do efeito da terapia de fotobiomodulação sobre a FM.

Contudo existe poucos estudos que investigue o intervalo ideal para a otimização do efeito da irradiação terapia de fotobiomodulação sobre a fadiga muscular. Demostrando assim, que ainda existe uma grande lacuna sobre esse parâmetro na literatura atua.

Em nossa pesquisa todos os estudos indicaram quais os músculos que foram irradiados pela terapia de fotobiomodulação em suas metodologias. Cerca de $100 \%$ utilizaram o quadríceps como objeto de estudo, $50 \%$ combinaram com a irradiação dos músculos Isquiotibiais e gastrocnêmicos.

Em relação a atividade escolhida para avaliar a FM e performance muscular, cerca de $40 \%$ dos estudos utilizaram o equipamento dinamômetro isocinético, seguido de $20 \%$ de utilização de salto, $20 \%$ testes de corrida e $10 \%$ teste em ciclismo.

Todos os estudos avaliados consideram positivo o efeito da irradiação da terapia de fotobiomodulação no atraso do processo de FM.

Demostrando assim, que atualmente, existem evidências crescentes que demonstram o potencial da terapia de fotobiomodulação como agente ergogênico, uma vez que essa ferramenta é capaz de melhorar o desempenho atlético, bem como melhorar recuperação pós-exercício (Tomazini et al., 2019).

\section{Considerações Finais}

A terapia de fotobiomodulação realizada por meio da utilização da TLBI e LEDT é capaz de atrasar o processo de FM e consequentemente aumentar a performance. Contudo os parâmetros ideais de aplicação dessa ferramenta ainda continuam demostrando muitas variáveis. Exceto pelo comprimento de onda, que é o único parâmetro de aplicação que demostra uma tendência, na qual, é difundido pela literatura que a irradiação no infravermelho possibilita um melhor efeito ergogênico no tecido muscular. Entretanto, os parâmetros como a energia total $(\mathrm{J})$ e o intervalo ainda não se têm um consenso difundo na literatura. Nesta premissa, é de suma importância de estudos clínicos que busquem sanar essas lacunas.

\section{Referências}

Albuquerque-Pontes, G. M., Vieira, R. P., Tomazoni, S. S., Caires, C. O., Nemeth, V., Vanin, A. A., Santos, L. A., Pinto, H. D., Marcos, R. L., Bjordal, J. M., de Carvalho, P., \& Leal-Junior, E. C. (2015). Effect of pre-irradiation with different doses, wavelengths, and application intervals of low-level laser therapy on cytochrome c oxidase activity in intact skeletal muscle of rats. Lasers in medical science, 30(1), 59-66. https://doi.org/10.1007/s10103-014-1616-2

Anders, J. J., Arany, P. R., Baxter, G. D., \& Lanzafame, R. J. (2019). Light-Emitting Diode Therapy and Low-Level Light Therapy Are Photobiomodulation Therapy. Photobiomodulation, photomedicine, and laser surgery, 37(2), 63-65. https://doi.org/10.1089/photob.2018.4600

Boccia, G., Dardanello, D., Zoppirolli, C., Bortolan, L., Cescon, C., Schneebeli, A., Vernillo, G., Schena, F., Rainoldi, A., \& Pellegrini, B. (2017). Central and peripheral fatigue in knee and elbow extensor muscles after a long-distance cross-country ski race. Scandinavian journal of medicine \& science in sports, 27(9), 945-955. https://doi.org/10.1111/sms.12718

Costa, D. R., Costa, D.R., Pessoa, D.R., Masulo, L.J., Arisawa, E. A. L.S., \& Nicolau, R.A. (2017) Effect of LED therapy on temporomandibular disorder: a case study. Sci. Med.;27: 258-272. 
Costa, D. R., Delpasso, C. A., Costa, D. R., \& Nicolau, R. A. (2015) Efeito da fototerapia de baixa intensidade na fadiga muscular em humanos - revisão de literatura. XV Encontro Latino-americano de Pós- Graduação - Universidade do Vale do Paraíba. http://www.inicepg.univap.br/cd/INIC_2015/anais/arquivos/RE_0676_1358_01.pdf

Costa, D. R., Pessoa, D. R., Seefeldt, V. B., Costa, D. R., Maia, D., Dos Santos Maciel, T., Mota, B., Delpasso, C. A., Ribeiro, C., \& Nicolau, R. A. (2021). Orofacial evaluation of individuals with temporomandibular disorder after LED therapy associated or not of occlusal splint: a randomized double-blind controlled clinical study. Lasers in medical science. https://doi.org/10.1007/s10103-021-03269-2

da Cunha, R. A., Pinfildi, C. E., de Castro Pochini, A., \& Cohen, M. (2020). Photobiomodulation therapy and NMES improve muscle strength and jumping performance in young volleyball athletes: a randomized controlled trial study in Brazil. Lasers in medical science, 35(3), 621-631. https://doi.org/10.1007/s10103-019-02858-6

de Oliveira, A. R., Vanin, A. A., Tomazoni, S. S., Miranda, E. F., Albuquerque-Pontes, G. M., De Marchi, T., Dos Santos Grandinetti, V., de Paiva, P., Imperatori, T., de Carvalho, P., Bjordal, J. M., \& Leal-Junior, E. (2017). Pre-Exercise Infrared Photobiomodulation Therapy (810 nm) in Skeletal Muscle Performance and Postexercise Recovery in Humans: What Is the Optimal Power Output?. Photomedicine and laser surgery, 35(11), 595-603. https://doi.org/10.1089/pho.2017.4343

Dellagrana, R. A., Rossato, M., Sakugawa, R. L., Baroni, B. M., \& Diefenthaeler, F. (2018). Photobiomodulation Therapy on Phy siological and Performance Parameters During Running Tests: Dose-Response Effects. Journal of strength and conditioning research, 32(10), 2807-2815. https://doi.org/10.1519/JSC.0000000000002488

Dornelles, M. P., Fritsch, C. G., Sonda, F. C., Johnson, D. S., Leal-Junior, E., Vaz, M. A., \& Baroni, B. M. (2019). Photobiomodulation therapy as a tool to prevent hamstring strain injuries by reducing soccer-induced fatigue on hamstring muscles. Lasers in medical science, 34(6), 1177-1184. https://doi.org/10.1007/s10103-018-02709-w

Ferraresi, C., Bertucci, D., Schiavinato, J., Reiff, R., Araújo, A., Panepucci, R., Matheucci, E., Jr, Cunha, A. F., Arakelian, V. M., Hamblin, M. R., Parizotto, N., \& Bagnato, V. (2016). Effects of Light-Emitting Diode Therapy on Muscle Hypertrophy, Gene Expression, Performance, Damage, and Delayed-Onset Muscle Soreness: Case-control Study with a Pair of Identical Twins. American journal of physical medicine \& rehabilitation, 95(10), 746-757. https://doi.org/10.1097/PHM.0000000000000490

Ferraresi, C., de Sousa, M. V., Huang, Y. Y., Bagnato, V. S., Parizotto, N. A., \& Hamblin, M. R. (2015). Time response of increases in ATP and muscle resistance to fatigue after low-level laser (light) therapy (LLLT) in mice. Lasers in medical science, 30(4), 1259-1267. https://doi.org/10.1007/s10103-015$1723-8$

Hemmings, T. J., Kendall, K. L., \& Dobson, J. L. (2017). Identifying Dosage Effect of Light-Emitting Diode Therapy on Muscular Fatigue in Quadriceps. Journal of strength and conditioning research, 31(2), 395-402. https://doi.org/10.1519/JSC.0000000000001523

Lanferdini, F. J., Krüger, R. L., Baroni, B. M., Lazzari, C., Figueiredo, P., Reischak-Oliveira, A., \& Vaz, M. A. (2018). Low-level laser therapy improves the VO2 kinetics in competitive cyclists. Lasers in medical science, 33(3), 453-460. https://doi.org/10.1007/s 10103-017-2347-y

Leal Junior, E., Bjordal, J., \& Lopes-Martins, R. (2010) A laserterapia de baixa potência melhora o desempenho muscular mensurado por dinamometria isocinética em humanos. Fisioterapia e Pesquisa. 17(4), 317-321.

Miranda, E. F., Tomazoni, S. S., de Paiva, P., Pinto, H. D., Smith, D., Santos, L. A., de Tarso Camillo de Carvalho, P., \& Leal-Junior, E. (2018). When is the best moment to apply photobiomodulation therapy (PBMT) when associated to a treadmill endurance-training program? A randomized, triple-blinded, placebo-controlled clinical trial. Lasers in medical science, 33(4), 719-727. https://doi.org/10.1007/s10103-017-2396-2

Moher, D., Liberati, A., Tetzlaff, J., Altman, D. G., \& PRISMA Group (2009). Preferred reporting items for systematic reviews and meta-analyses: the PRISMA statement. PLoS medicine, 6(7), e1000097. https://doi.org/10.1371/journal.pmed.1000097

Orssatto, L., Detanico, D., Kons, R. L., Sakugawa, R. L., da Silva, J. N., Jr, \& Diefenthaeler, F. (2019). Photobiomodulation Therapy Does Not Attenuate Fatigue and Muscle Damage in Judo Athletes: A Randomized, Triple-Blind, Placebo-Controlled Trial. Frontiers in physiology, 10, 811. https://doi.org/10.3389/fphys.2019.00811

Pessoa, D. R., Costa, D. R., Prianti, B. M., Costa, D. R., Delpasso, C. A., Arisawa, E., \& Nicolau, R. A. (2018). Association of facial massage, dry needling, and laser therapy in Temporomandibular Disorder: case report. CoDAS, 30(6), e20170265. https://doi.org/10.1590/2317-1782/20182017265

Rossato, M., Dellagrana, R. A., Sakugawa, R. L., Lazzari, C. D., Baroni, B. M., \& Diefenthaeler, F. (2018). Time Response of Photobiomodulation Therapy on Muscular Fatigue in Humans. Journal of strength and conditioning research, 32(11), 3285-3293. https://doi.org/10.1519/JSC.0000000000002339

Tomazoni, S. S., Machado, C., De Marchi, T., Casalechi, H. L., Bjordal, J. M., de Carvalho, P., \& Leal-Junior, E. (2019). Infrared Low-Level Laser Therapy (Photobiomodulation Therapy) before Intense Progressive Running Test of High-Level Soccer Players: Effects on Functional, Muscle Damage, Inflammatory, and Oxidative Stress Markers-A Randomized Controlled Trial. Oxidative medicine and cellular longevity, $2019,6239058$. https://doi.org/10.1155/2019/6239058 